\title{
GENUS, THICKNESS AND CROSSING NUMBER OF GRAPHS ENCODING THE GENERATING PROPERTIES OF FINITE GROUPS
}

\author{
CRISTINA ACCIARRI AND ANDREA LUCCHINI
}

\begin{abstract}
Assume that $G$ is a finite group and let $a$ and $b$ be non-negative integers. We define an undirected graph $\Gamma_{a, b}(G)$ whose vertices correspond to the elements of $G^{a} \cup G^{b}$ and in which two tuples $\left(x_{1}, \ldots, x_{a}\right)$ and $\left(y_{1}, \ldots, y_{b}\right)$ are adjacent if and only $\left\langle x_{1}, \ldots, x_{a}, y_{1}, \ldots, y_{b}\right\rangle=G$. Our aim is to estimate the genus, the thickness and the crossing number of the graph $\Gamma_{a, b}(G)$ when $a$ and $b$ are positive integers.
\end{abstract}

\section{INTRODUCTION}

Generating sets of a finite group may be quite complicated. If a group $G$ is $d$-generated, the question of which sets of $d$ elements of $G$ generate $G$ is nontrivial. The simplest interesting case is when $G$ is 2 -generated. One tool developed to study generators of 2-generated finite groups is the generating graph $\Gamma(G)$ of $G$; this is the graph which has the elements of $G$ as vertices and an edge between two elements $g_{1}$ and $g_{2}$ if $G$ is generated by $g_{1}$ and $g_{2}$. Note that the generating graph may be defined for any group, but it only has edges if $G$ is 2 -generated. A wider family of graphs which encode the generating property of $G$ when $G$ is an arbitrary finite group was introduced and investigated in 11. The definition of these graphs is the following. Assume that $G$ is a finite group and let $a$ and $b$ be non-negative integers. We define an undirected graph $\Gamma_{a, b}(G)$ whose vertices correspond to the elements of $G^{a} \cup G^{b}$ and in which two tuples $\left(x_{1}, \ldots, x_{a}\right)$ and $\left(y_{1}, \ldots, y_{b}\right)$ are adjacent if and only $\left\langle x_{1}, \ldots, x_{a}, y_{1}, \ldots, y_{b}\right\rangle=G$. Notice that $\Gamma_{1,1}(G)$ is the generating graph of $G$, so these graphs can be viewed as a natural generalization of the generating graph.

Let $\Delta$ be a graph. The genus $\gamma(\Delta)$ of $\Delta$ is the minimum integer $g$ such that there exists an embedding of $\Delta$ into the orientable surface $S_{g}$ of genus $g$ (or in other words the minimum number $g$ of handles which must be added to a sphere so that $\Delta$ can be embedded on the resulting surface). The thickness $\theta(\Delta)$ of $\Delta$ is the minimum number of planar graphs into which the edges of $\Delta$ can be partitioned. The crossing number $\operatorname{cr}(\Delta)$ of $\Delta$ is the minimum number of crossings in any simple drawing of $\Delta(G)$. In this paper we investigate genus, thickness and crossing number of the graphs $\Gamma_{a, b}(G)$, when $1 \leq a \leq b$ and $a+b \geq d(G)$, where $d(G)$ is the smallest cardinality of a generating set of $G$. Notice that the case $a=0$ is not interesting: the graph $\Gamma_{0, b}(G)$ is a star with an internal node corresponding to the empty set and with $\phi_{G}(b)$ leaves, being $\phi_{G}(b)$ be the number of the generating $b$-uples of $G$. Our main result is the following:

Key words and phrases. generating graph; genus; thickness; crossing numbers. 
Theorem 1. Assume that $G$ is a nontrivial d-generated finite group and that $a, b$ are positive integer with $a+b \geq d$. Then

$$
\begin{aligned}
\gamma\left(\Gamma_{a, b}(G)\right) & \geq \frac{|G|^{b}}{6}\left(\frac{\sqrt{|G|}}{16}-3\right), \\
\theta\left(\Gamma_{a, b}(G)\right) & \geq \frac{\sqrt{|G|}}{48}, \\
\operatorname{cr}\left(\Gamma_{a, b}(G)\right) & \geq \frac{|G|^{d+\frac{1}{2}}}{29}\left(\frac{1}{2^{11}}-\frac{70}{|G|^{3 / 2}}\right) .
\end{aligned}
$$

In order to estimate $\gamma\left(\Gamma_{a, b}(G)\right), \theta\left(\Gamma_{a, b}(G)\right)$ and $\operatorname{cr}\left(\Gamma_{a, b}(G)\right)$, it is important to obtain a lower bound for the ratio $e\left(\Gamma_{a, b}(G)\right) / v\left(\Gamma_{a, b}(G)\right)$ between the number of edges and the number of vertices of the graph $\Gamma_{a, b}(G)$. We will see in Section 3 , that this is essentially related to the estimation of the ratio $\phi_{G}(d) /|G|^{d-1}$ for a $d$-generated finite group. Our main result in this direction is the following.

Theorem 2. If $G$ is a d-generated finite group, then

$$
\frac{\phi_{G}(d)}{|G|^{d-1}} \geq \frac{\sqrt{|G|}}{2} .
$$

We think that this is a result of independent interest. For example it implies the following corollary.

Corollary 3. Let $G$ be a finite group and let $d=d(G)$. Denote by $\rho(G)$ the number of elements $g$ in $G$ such that $G=\left\langle g, x_{1}, \ldots, x_{d-1}\right\rangle$, for some $x_{1}, \ldots, x_{d-1} \in G$. We have

$$
\rho(G) \geq \frac{|G|^{1-\frac{1}{2 d}}}{2^{\frac{1}{d}}}
$$

Recall that a graph is said to be embeddable in the plane, or planar, if it can be drawn in the plane so that its edges intersect only at their ends. In [12] a classification of the 2-generated finite groups with planar generating graph is given. We generalize this result as follows.

Theorem 4. Let $G$ be a nontrival finite group and let $a$ and $b$ be two positive integers with $a \leq b$ and $a+b \geq d(G)$. Then $\Gamma_{a, b}(G)$ is planar if and only if one of the following occurs:

(1) $G \in\left\{C_{3}, C_{4}, C_{6}, C_{2} \times C_{2}, D_{3}, D_{4}, Q_{8}, C_{4} \times C_{2}, D_{6}\right\}$ and $(a, b)=(1,1)$.

(2) $G \cong C_{2}$ and either $a=1$ or $(a, b)=(2,2)$.

\section{Proof of Theorem 2}

Let $G$ be a $d$-generated finite group and let $\phi_{G}(d)$ denote the number of the generating $d$-uples $\left(g_{1}, \ldots, g_{d}\right) \in G^{d}$ with $\left\langle g_{1}, \ldots, g_{d}\right\rangle=G$. Clearly $P_{G}(d)=\phi_{G}(d) /|G|^{d}$ coincides with the probability that $d$ randomly chosen elements from $G$ generate $G$.

Definition 5. For a d-generated finite group $G$, set

$$
\alpha(G, d):=\frac{\phi_{G}(d)}{|G|^{d-1}}=P_{G}(d)|G| .
$$


Let $N$ be a normal subgroup of a finite group $G$ and choose $g_{1}, \ldots, g_{k} \in G$ with the property that $G=\left\langle g_{1}, \ldots, g_{k}\right\rangle N$. By a result of Gaschütz [8] the cardinality of the set

$$
\Phi_{N}\left(g_{1}, \ldots, g_{k}\right)=\left\{\left(n_{1}, \ldots, n_{k}\right) \in N \mid\left\langle g_{1} n_{1}, \ldots, g_{k} n_{k}\right\rangle=G\right\}
$$

does not depend on the choice of $g_{1}, \ldots, g_{k}$. Let

$$
P_{G, N}(k)=\frac{\left|\Phi_{N}\left(g_{1}, \ldots, g_{k}\right)\right|}{|N|^{k}} .
$$

Notice that if $k \geq d(G / N)$, then $P_{G, N}(k)=P_{G}(k) / P_{G / N}(k)$.

Definition 6. Let $N$ be a normal subgroup of a d-generated finite group $G$. Set

$$
\alpha(G, N, d):=\frac{\alpha(G, d)}{\alpha(G / N, d))}=P_{G, N}(d)|N| .
$$

Lemma 7. Assume that $N$ is a minimal abelian normal subgroup of a d-generated finite group $G$. We have $|N|=p^{a}$, where $p$ is a prime and $a$ is a positive integer. Let $c$ be the number of complements of $N$ in $G$. Then

$$
\alpha(G, N, d)=\frac{p^{d \cdot a}-c}{p^{(d-1) \cdot a}} \geq p^{a}-p^{a-1}=p^{a-1}(p-1) .
$$

In particular

(1) $\alpha(G, N, d)=1$ if and only if $|N|=2, N$ has a complement in $G$ and $G / N$ admits $C_{2}^{d-1}$ as an epimorphic image.

(2) $\alpha(G, N, d) \geq 3 / 2$ if $|N|=2, N$ has a complement in $G$ and $C_{2}^{d-1}$ is not an epimorphic image of $G / N$.

(3) $\alpha(G, N, d) \geq 2$ in all the remaining cases.

Proof. By [9, Satz 2], $P_{G, N}(d)=1-c / p^{d \cdot a}$, hence $\alpha(G, N, d)=\frac{p^{d \cdot a}-c}{p^{(d-1) \cdot a}}$. If $c \neq 0$, then $c$ is the order of the group $\operatorname{Der}(G / N, N)$ of derivations from $G / N$ to $N$; in particular $c$ is a power of $p$. Moreover, since $G$ is $d$-generated, it must be $c<p^{d \cdot a}$ and consequently

$$
\alpha(G, N, d)=\frac{p^{d \cdot a}-c}{p^{(d-1) \cdot a}} \geq \frac{p^{d \cdot a}-p^{d \cdot a-1}}{p^{(d-1) \cdot a}}=p^{a}-p^{a-1} .
$$

In particular we can have $\alpha(G, N)<2$ only if $|N|=2$ and $c \neq 0$. Let $H$ be a complement of $N$ in $G$ and let $K=H^{\prime} H^{2}$. We have $c=|\operatorname{Der}(H, N)|=|\operatorname{Hom}(H / K, N)|$. Since $G$ is $d$-generated, we have $H / K \cong C_{2}^{t}$ with $t<d$. We have $c=2^{t}$ and $\alpha(G, N, d)=2-2^{t-d+1}$.

If a group $G$ acts on a group $A$ via automorphisms, then we say that $A$ is a $G$-group. If $G$ does not stabilise any nontrivial proper subgroup of $A$, then $A$ is called an irreducible $G$-group. Two $G$-groups $A$ and $B$ are said to be $G$ isomorphic, or $A \cong_{G} B$, if there exists a group isomorphism $\phi: A \rightarrow B$ such that $\phi(g(a))=g(\phi(a))$ for all $a \in A, g \in G$. Following [11, we say that two $G$-groups $A$ and $B$ are $G$-equivalent and we put $A \equiv_{G} B$, if there are isomorphisms $\phi: A \rightarrow B$ and $\Phi: A \rtimes G \rightarrow B \rtimes G$ such that the following diagram commutes:

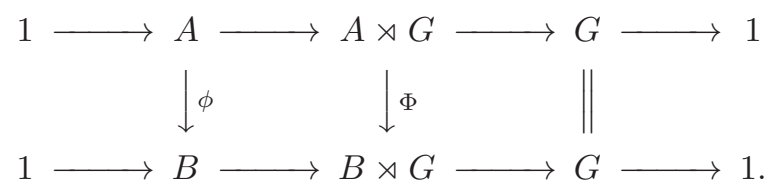


Note that two $G$-isomorphic $G$-groups are $G$-equivalent. In the abelian case, the converse is true: if $A_{1}$ and $A_{2}$ are abelian and $G$-equivalent, then $A_{1}$ and $A_{2}$ are also $G$-isomorphic. It is known (see for example [11, Proposition 1.4]) that two chief factors $A_{1}$ and $A_{2}$ of $G$ are $G$-equivalent if and only if either they are $G$-isomorphic, or there exists a maximal subgroup $M$ of $G$ such that $G / \operatorname{Core}_{G}(M)$ has two minimal normal subgroups, $N_{1}$ and $N_{2}, G$-isomorphic to $A_{1}$ and $A_{2}$ respectively. Let $A=$ $X / Y$ be a chief factor of $G$. We say that $A=X / Y$ is a Frattini chief factor if $X / Y$ is contained in the Frattini subgroup of $G / Y$; this is equivalent to saying that $A$ is abelian and there is no complement to $A$ in $G$. The number of non-Frattini chief factors $G$-equivalent to $A$ in any chief series of $G$ does not depend on the series, and so this number is well-defined: we will denote it by $\delta_{A}(G)$.

The following numerical results will be useful.

Lemma 8. [7, 9.15 p. 54] Let $n>0$, then

$$
\sqrt{2 \pi} \cdot n^{n+\frac{1}{2}} \cdot e^{-n} \cdot e^{\frac{1}{12 n+1}} \leq n ! \leq \sqrt{2 \pi} \cdot n^{n+\frac{1}{2}} \cdot e^{-n} \cdot e^{\frac{1}{12 n}}
$$

Corollary 9. If $t<n$, then

$$
\frac{n !}{(n-t) !} \geq \frac{9}{10} \frac{n^{t}}{e^{t}}
$$

Proof.

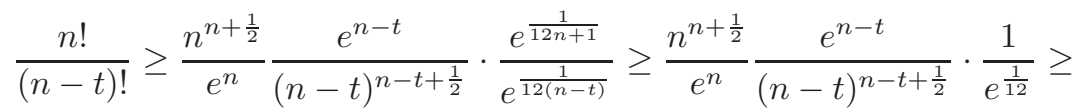

$$
\begin{aligned}
& \geq \frac{n^{n+\frac{1}{2}}}{e^{n}} \frac{e^{n-t}}{(n-t)^{n-t+\frac{1}{2}}} \cdot \frac{9}{10} \geq \frac{9}{10} \frac{n^{n+\frac{1}{2}}}{(n-t)^{n-t+\frac{1}{2}}} \cdot \frac{1}{e^{t}} \geq \\
& \geq \frac{9}{10} \frac{n^{n+\frac{1}{2}}}{n^{n-t+\frac{1}{2}}} \cdot \frac{1}{e^{t}}=\frac{9}{10} \frac{n^{t}}{e^{t}} .
\end{aligned}
$$

Proposition 10. Let $G$ be a finite group and let $B$ be a non-abelian chief factor of $G$. Denote by $t=\delta_{G}(B)$ the number of factors $G$-equivalent to $B$ in a given chief series of $G$. More precisely let $X_{1} / Y_{1}, X_{2} / Y_{2}, \ldots, X_{t} / Y_{t}$, with $Y_{t} \leq X_{t} \leq$ $\cdots \leq Y_{1} \leq X_{1}$, be the factors $G$-equivalent to $B$ in a given chief series of $G$. For $1 \leq i \leq t$, let $\alpha_{i}=\alpha\left(G / Y_{i}, X_{i} / Y_{i}, d\right)$. We have

$$
\prod_{1 \leq i \leq t} \alpha_{i} \geq \frac{9}{10}\left(\frac{53|B|}{90 e}\right)^{t}
$$

Proof. Let $L=G / C_{G}(B)$ be the monolithic primitive group associated to $B$ and assume $L=\left\langle l_{1}, \ldots, l_{d}\right\rangle$. Moreover define $\Gamma:=C_{\text {Aut }(B)}(L / B)|, \gamma=| \Gamma \mid, \Phi:=$ $\Phi_{B}\left(l_{1}, \ldots, l_{d}\right)$. By [5, Proposition 16], for $1 \leq i \leq t$, we have

$$
\alpha_{i}=\frac{|\Phi|}{|B|^{d-1}}-\frac{(i-1) \gamma}{|B|^{d-1}} .
$$

Let $\rho=|\Phi| / \gamma$ (notice that $\rho$ is an integer) and let $\tau=|B|^{d-1} / \gamma$. It follows from [6], Theorem 1.1] that $\rho / \tau \geq \frac{53}{90}|B|$. In view of Corollary 9 we have

$$
\prod_{1 \leq i \leq t} \alpha_{i}=\frac{\rho(\rho-1) \cdots(\rho-(t-1))}{\tau^{t}} \geq \frac{9}{10 \cdot e^{t}}\left(\frac{\rho}{\tau}\right)^{t} \geq \frac{9}{10}\left(\frac{53|B|}{90 e}\right)^{t} .
$$


Next we deal with the proof of Theorem 2

Proof of Theorem 2, Let $X_{t} \leq X_{t-1} \leq \cdots \leq X_{1}=G$ be a chief series of $G$ and for $1 \leq i \leq t-1$, let $\alpha_{i}=\alpha\left(G / X_{i+1}, X_{i} / X_{i+1}, d\right)$. Since $d(G)=d$, it must be $\delta_{G}\left(C_{2}\right) \leq \bar{d}$ and this implies in particular that there exists at most a unique index $j^{*}$ such that $X_{j^{*}} / X_{j^{*}+1}$ has order 2 , is complemented in $G / X_{j^{*}+1}$ and the quotient $G / X_{j^{*}}$ admits $C_{2}^{d-1}$ as an epimorphic image. If $\left|X_{i} / X_{i+1}\right|=2$ and $i \neq j^{*}$, then, by Lemma 7, $\alpha_{i} \geq 3 / 2 \geq \sqrt{2}=\sqrt{\left|X_{i} / X_{i+1}\right|}$. If $X_{i} / X_{i+1}$ is abelian and $\left|X_{i} / X_{i+1}\right|=$ $p_{i}^{n_{i}}>2$, then, again by Lemma 7, $\alpha_{i} \geq p_{i}^{n_{i}-1}\left(p_{i}-1\right) \geq \sqrt{p_{i}^{n_{i}}}=\sqrt{\left|X_{i} / X_{i+1}\right|}$. Now assume that $B$ is a non-abelian chief factor of $G$ and let

$$
I_{B}=\left\{1 \leq k \leq t-1 \mid X_{k} / X_{k+1} \equiv_{G} B\right\} .
$$

By Proposition 10, noticing that $\delta_{B}(G)=\left|I_{B}\right|$ and $|B| \geq 6 \sqrt{|B|}$ since $|B| \geq 60$, we have

$$
\begin{aligned}
\prod_{k \in I_{B}} \alpha_{k} & \geq \frac{9}{10}\left(\frac{53|B|}{90 e}\right)^{\delta_{B}(G)} \geq\left(\frac{53|B|}{100 e}\right)^{\delta_{B}(G)} \geq \\
& \geq\left(\frac{|B|}{6}\right)^{\delta_{B}(G)} \geq(\sqrt{|B|})^{\delta_{B}(G)}=\prod_{k \in I_{B}} \sqrt{\left|X_{k} / X_{k+1}\right|} .
\end{aligned}
$$

The result follows since $\alpha(G, d)=\prod_{1 \leq i \leq t-1} \alpha_{i}$ and $|G|=\prod_{1 \leq i \leq t-1}\left|X_{i} / X_{i+1}\right|$.

We close this section with the proof of Corollary 3 .

Proof of Corollary [3. By Theorem [2,

$$
\rho(G)^{d} \geq \phi_{G}(d)=\alpha(G, d)|G|^{d-1} \geq \frac{|G|^{\frac{1}{2}}|G|^{d-1}}{2}=\frac{|G|^{d-\frac{1}{2}}}{2} .
$$

\section{Proof of Theorem 1}

Before proving Theorem 1, we recall some general results in graph theory concerning lower bounds for the genus, the thickness and the crossing number of a simple graph $\Delta$.

Proposition 11. [10, 7.2.4 - F35] If $\Delta$ is a simple graph with e edges and $v$ vertices, then

$$
\gamma(\Delta) \geq 1-\frac{v}{2}+\frac{e}{6} \geq \frac{v}{6}\left(\frac{e}{v}-3\right)
$$

Proposition 12. [3, 10.3.6 (a)]. If $\Delta$ is a simple graph with e edges and $v \geq 3$ vertices, then

$$
\theta(\Delta) \geq \frac{e}{3 v-6}
$$

Proposition 13. [2, Theorem 6] If $\Delta$ is a simple graph with e edges and $v$ vertice, then

$$
\operatorname{cr}(\Delta) \geq \frac{e^{3}}{29 v^{2}}-\frac{35}{29} v
$$

Assume that $G$ is a finite group and let $a$ and $b$ be positive integers. Let $d=a+b \geq d(G)$. If $a \neq b$ then $\Gamma_{a, b}(G)$ is a bipartite graphs with two parts, one corresponding to the elements of $G^{a}$ and the other to the elements of $G^{b}$. In particular $\Gamma_{a, b}(G)$ has $|G|^{a}+|G|^{b}$ vertices and there exists a bijective correspondence between the set of the generating $d$-uples of $G$ and the set of the edges 
of $\Gamma_{a, b}(G)$ : indeed if $\left\langle g_{1}, \ldots, g_{d}\right\rangle=G$, then $\left(g_{1}, \ldots, g_{a}\right)$ and $\left(g_{a+1}, \ldots, g_{d}\right)$ are adjacent vertices of the graph. Hence the number of edges of $\Gamma_{a, b}(G)$ is $\phi_{G}(d)$. The situation is different if $a=b$. In that case $\Gamma_{a, a}(G)$ has $|G|^{a}$ vertices, $\phi_{G}(a)$ loops and other $\left(\phi_{G}(d)-\phi_{G}(a)\right) / 2$ edges connecting two different vertices (in other words if $e$ is the the number of edges, excluding the loops, and $l$ is the number of loops, then $\left.2 e+l=\phi_{G}(d)\right)$; indeed the two elements $\left(g_{1}, \ldots, g_{a}, g_{a+1}, \ldots, g_{d}\right)$ and $\left(g_{a+1}, \ldots, g_{d}, g_{1}, \ldots, g_{a}\right)$ give rise to the same edge in $\Gamma_{a, a}(G)$. Summarizing, let $\nu$ and $\eta$ be, respectively, the number of vertices and edges of $\Gamma_{a, b}(G)$, excluding the loops. We have

$$
|G|^{b} \leq \nu \leq|G|^{a}+|G|^{b} \leq 2|G|^{d-1}
$$

Moreover $\eta=\phi_{G}(a+b)$ if $a \neq b, \eta=\left(\phi_{G}(2 a)-\phi_{G}(a)\right) / 2$ if $a=b$. If $\phi_{G}(a) \neq 0$, then $\phi_{G}(2 a) \geq \phi_{G}(a)|G|^{a}$, so $\phi_{G}(a) \leq \phi_{G}(2 a) /|G|^{a}$. So if $|G| \geq 2$, then $\eta \geq \phi_{G}(d) / 4$. By applying Theorem 2 and Propositions 1112 and 13 respectively it follows that if $G \neq 1$, then we have the following inequalities.

$$
\begin{aligned}
\gamma\left(\Gamma_{a, b}(G)\right) \geq & \frac{\nu}{6}\left(\frac{\eta}{\nu}-3\right) \geq \frac{|G|^{b}}{6}\left(\frac{\phi_{G}(d)}{8|G|^{d-1}}-3\right) \geq \frac{|G|^{b}}{6}\left(\frac{\sqrt{|G|}}{16}-3\right) \\
& \theta\left(\Gamma_{a, b}(G)\right) \geq \frac{\eta}{3 \nu} \geq \frac{\phi_{G}(d)}{24|G|^{d-1}} \geq \frac{\sqrt{|G|}}{48} \\
\operatorname{cr}\left(\Gamma_{a, b}(G)\right) \geq & \frac{\eta^{3}}{29 \cdot \nu^{2}}-\frac{35}{29} \cdot \nu \geq \frac{\left(\phi_{G}(d)\right)^{3}}{29 \cdot 4^{3} \cdot 4 \cdot\left(|G|^{d-1}\right)^{2}}-\frac{70 \cdot|G|^{d-1}}{29} \\
\geq & \frac{\phi_{G}(d)|G|}{29 \cdot 4^{5}}-\frac{70 \cdot|G|^{d-1}}{29} \geq \frac{|G|^{d+\frac{1}{2}}}{29 \cdot 2^{11}}-\frac{70 \cdot|G|^{d-1}}{29} .
\end{aligned}
$$

This concludes the proof of Theorem 1

\section{Proof of Theorem 4}

The main goal of this section is to prove Theorem 4. We star with two preliminary results.

Proposition 14. 4, Lemma 9.23]. A simple bipartite planar graph on v vertices, whose every connected component contains at least three vertices, can have not more than $2 v-4$ edges.

Lemma 15. Let $G$ be a finite group and let $b \geq d(G)$. Consider the set $W=$ $\left\{\left(x_{1}, \ldots, x_{b}\right) \in G^{b} \mid\left\langle x_{1}, \ldots, x_{b}\right\rangle=G\right\}$. If $G$ is not cyclic, then $|W| \geq 3$.

Proof. Assume $d=d(G)$ and $G=\left\langle g_{1}, \ldots, g_{d}\right\rangle$. Then $\left(g_{1}, g_{2}, g_{3}, \ldots, g_{d}, 1, \ldots, 1\right)$, $\left(g_{1} g_{2}, g_{2}, g_{3}, \ldots, g_{d}, 1, \ldots, 1\right)$ and $\left(g_{1}, g_{1} g_{2}, g_{3}, \ldots, g_{d}, 1, \ldots, 1\right)$ are three different elements of $W$.

We are now ready to embark on the proof of Theorem 4 .

Proof of Theorem 4. Let $a$ and $b$ be positive integers with $a+b \geq d(G)$. We want to discuss when $\Gamma_{a, b}(G)$ is planar. We assume $a+b \geq d(G)$ and $a \leq b$. If $a=0$, then $\Gamma_{a, b}(G)$ is a star, so it is planar. We may exclude from our discussion the case $a=b=1$, since the result in this case follows from the main result in [12] (notice that the cyclic group $C_{5}$ appears in the statement of [12, Theorem 1.1] but not in the statement of Theorem [4] this is because in [12] the identity element is not included in the vertex-set of $\left.\Gamma_{1,1}(G)\right)$.

First assume that $G=\langle g\rangle$ is cyclic. 
- If $a \geq 3$, take

$\alpha_{1}=(1,1, g, 1, \ldots, 1), \alpha_{2}=(1, g, g, 1, \ldots, 1), \alpha_{3}=(1, g, 1,1, \ldots, 1) \in G^{a}$,

$\beta_{1}=(g, 1, g, 1, \ldots, 1), \beta_{2}=(g, g, g, 1, \ldots, 1), \beta_{3}=(g, g, 1,1, \ldots, 1) \in G^{b}$.

- If $a=2$ and $|G| \neq 2$, take

$\alpha_{1}=(1, g), \alpha_{2}=(g, 1), \alpha_{3}=(g, g) \in G^{2}$,

$\beta_{1}=\left(1, g^{2}, 1, \ldots, 1\right), \beta_{2}=\left(g^{2}, 1, \ldots, 1\right), \beta_{3}=\left(g^{2}, g^{2}, 1, \ldots, 1\right) \in G^{b}$.

- If $a=2$ and $|G|=2$ and $b \geq 3$, take

$\alpha_{1}=(1, g), \alpha_{2}=(g, 1), \alpha_{3}=(g, g) \in G^{2}$,

$\beta_{1}=(1, g, g, 1 \ldots, 1), \beta_{2}=(g, 1, g, 1 \ldots, 1), \beta_{3}=(g, g, g, 1 \ldots, 1) \in G^{b}$.

In all these cases, since $\alpha_{i}$ and $\beta_{j}$ are adjacent for every $1 \leq i, j \leq 3, \Gamma_{a, b}(G)$ contains $K_{3,3}$, so it is not planar. If $a=b=2$ and $|G|=2$, then $\Gamma_{2,2}(G) \cong K_{4}$ is planar. If $a=1$ and $|G|>2$, then we may consider the subgraph of $\Gamma_{1, b}(G)$ induced by the following vertices: $(1),(g),\left(g^{2}\right),(g, x, \ldots, x) \in G^{b}$ for $x \in G$. This subgraph is bipartite with $3+|G|$ vertices and $3|G|$ egdes. Since $3|G|>2(3+|G|)-4$, it follows from Proposition [14, that this graph is not planar. On the other hand, if $a=1$ and $|G|=2$, then it can be easily seen that the graph $\Gamma_{1, b}(G)$ is planar.

Now assume that $G$ is not cyclic. Let $d=d(G)$ and $G=\left\langle g_{1}, \ldots, g_{d}\right\rangle$.

First assume that $a \geq 2$. If $a+b=d$, then set

$$
\begin{aligned}
& \alpha_{1}=\left(g_{1}, g_{2}, g_{3}, \ldots, g_{a}\right) \in G^{a}, \\
& \alpha_{2}=\left(g_{1}, g_{1} g_{2}, g_{3}, \ldots, g_{a}\right) \in G^{a}, \\
& \alpha_{3}=\left(g_{1} g_{2}, g_{2}, g_{3}, \ldots, g_{a}\right) \in G^{a}, \\
& \beta_{1}=\left(g_{a+1}, g_{a+2}, g_{a+3}, \ldots, g_{b}\right) \in G^{b}, \\
& \beta_{2}=\left(g_{a+1} g_{a+2}, g_{a+2}, g_{a+3}, \ldots, g_{b}\right) \in G^{b}, \\
& \beta_{3}=\left(g_{a+1}, g_{a+1} g_{a+2}, g_{a+3}, \ldots, g_{b}\right) \in G^{b} .
\end{aligned}
$$

If $a+b>d$, choose three different elements $x, y, z$ of $G$ and set

$$
\begin{aligned}
\alpha_{1} & =\left(g_{1}, g_{2}, g_{3}, \ldots, g_{a}\right) \in G^{a}, \\
\alpha_{2} & =\left(g_{1}, g_{1} g_{2}, g_{3}, \ldots, g_{a}\right) \in G^{a}, \\
\alpha_{3} & =\left(g_{1} g_{2}, g_{2}, g_{3}, \ldots, g_{a}\right) \in G^{a}, \\
\beta_{1} & =\left(g_{a+1}, g_{a+2}, g_{a+3}, \ldots, g_{b}, x, \ldots, x\right) \in G^{b}, \\
\beta_{2} & =\left(g_{a+1}, g_{a+2}, g_{a+3}, \ldots, g_{b}, y, \ldots, y\right) \in G^{b}, \\
\beta_{3} & =\left(g_{a+1}, g_{a+2}, g_{a+3}, \ldots, g_{b}, z, \ldots, z\right) \in G^{b} .
\end{aligned}
$$

In both cases, since $\alpha_{i}$ and $\beta_{j}$ are adjacent for every $1 \leq i, j \leq 3, \Gamma_{a, b}(G)$ contains $K_{3,3}$, so it is not planar.

Assume $a=1$ and $a+b>d$. Let $W=\left\{\left(x_{1}, \ldots, x_{b}\right) \in G^{b} \mid\left\langle x_{1}, \ldots, x_{b}\right\rangle=G\right\}$ and let $x, y, z$ be three different elements of $G$. We may consider the subgraph of $\Gamma_{1, b}(G)$ induced by following vertices: $(x),(y),(z), w \in W$. This subgraph is bipartite with $3+|W|$ vertices and $3|W|$ egdes. Since, by Lemma 15 , $|W| \geq 3$, it 
follows $3|W|>2(3+|W|)-4$, and consequently, by Proposition 14 this graph is not planar.

Finally assume $a=1$ and $a+b=d$. Let $H=\left\langle g_{2}, \ldots, g_{b}\right\rangle$. If $H$ is cyclic, then $d(G) \leq 2$, in contradiction with $d(G)=1+b$ and $b>1$. Let $x, y, z$ be three different elements of $H$ and $W=\left\{\left(x_{1}, \ldots, x_{b}\right) \in G^{b} \mid\left\langle x_{1}, \ldots, x_{b}\right\rangle=H\right\}$. We may consider the subgraph of $\Gamma_{1, b}(G)$ induced by following vertices: $\left(g_{1} x\right),\left(g_{1} y\right),\left(g_{1} z\right), w \in W$. It is bipartite with $3+|W|$ vertices and $3|W|$ egdes. Since $H$ is not cyclic, we have $|W| \geq 3$ by Lemma 15. It follows $3|W|>2(3+|W|)-4$, and consequently, by Proposition 14, this graph is not planar.

\section{REFERENCES}

1. C. Acciarri and A. Lucchini, Graphs encoding the generating properties of a finite group, Mathematische Nachrichten, to appear. arXiv:1707.08348

2. E. Ackerman On topological graphs with at most four crossings per edge, Comput. Geom. 85 (2019), 101574, $31 \mathrm{pp}$

3. J. A. Bondy and U. S. R. Murty, Graph theory, Graduate Texts in Mathematics, 244. Springer, New York, 2008.

4. M. Cygan, F. Fomin, L. Kowalik, D. Lokshtanov, D. Marx, M. Pilipczuk, M. Pilipczuk, S. Saurabh, Parameterized algorithms. Springer, Cham, 2015 xviii+613

5. E. Detomi and A. Lucchini, Crowns and factorization of the probabilistic zeta function of a finite group, J. Algebra 265 (2003), no. 2, 651-668.

6. E. Detomi and A. Lucchini, Probabilistic generation of finite groups with a unique minimal normal subgroup, J. Lond. Math. Soc. (2) 87 (2013), no. 3, 689-706.

7. W. Feller, An introduction to probability theory and its applications. Vol. I. Third edition John Wiley \& Sons, Inc., New York-London-Sydney 1968 xviii+509 pp.

8. W. Gaschütz, $\mathrm{Zu}$ einem von B.H. und H. Neumann gestellten Problem, Mathematische Nachrichten 14 (1955), 249-252.

9. W. Gaschütz, Die Eulersche Funktion endlicher auflösbarer Gruppen, Illinois J. Math. 3 (1959), 469-476.

10. Handbook of graph theory. Second edition. Edited by J. Gross, J. Yellen and P. Zhang. Discrete Mathematics and its Applications (Boca Raton). CRC Press, Boca Raton, FL, 2014. xx+1610

11. P. Jiménez-Seral and J. Lafuente, On complemented non-abelian chief factors of a finite group. Israel J. Math. 106 (1998), 177-188.

12. A. Lucchini, Finite groups with planar generating graph, Australas. J. Combin. 76 (2020), part 1, 220-225.

Cristina Acciarri, Department of Mathematics, University of Brasilia, 70910-900

Brasília DF, BraZiL, EMAIL:ACCIARRICRISTINA@YAHOO.IT

Andrea Lucchini, Università degli Studi di Padova, Dipartimento di Matematica "Tullio Levi-Civita", Via Trieste 63, 35121 Padova, Italy, email: Lucchini@math.unipd.it 